\title{
Case Report ex Vivo Oocyte Collection to Maintain Fertility in a Patient with Micropapillary Serous Borderline Ovarian Tumor
}

\author{
Jitka Rezacova ${ }^{1}$, Petr Safar ${ }^{1}$, Lucie Petrouskova1 ${ }^{1}$, Blanka Koubkova ${ }^{1}$, Josef Plihal ${ }^{1}$, \\ Jana Rutarova1, Renata Valesova1, Katerina Dohnalova1, Jakub Rezac², Pavel Darebny', \\ Marketa Bahnikova' ${ }^{1}$, Jaroslav Feyereisl ${ }^{1}$ \\ ${ }^{1}$ Institute for the Care of Mother and Child, Prague, Czech Republic \\ ${ }^{2}$ Department of Urology, First Faculty of Medicine, Charles University and General University Hospital, \\ Prague, Czech Republic \\ Email: *jitka.rezacova@upmd.eu
}

How to cite this paper: Rezacova, J., Safar, P., Petrouskova, L., Koubkova, B., Plihal, J., Rutarova, J., Valesova, R., Dohnalova, K., Rezac, J., Darebny, P., Bahnikova, M. and Feyereisl, J. (2021) Case Report ex Vivo Oocyte Collection to Maintain Fertility in a Patient with Micropapillary Serous Borderline Ovarian Tumor. Advances in Reproductive Sciences, 9, 160-169. https://doi.org/10.4236/arsci.2021.92016

Received: March 3, 2021

Accepted: May 28, 2021

Published: May 31, 2021

Copyright (อ 2021 by author(s) and Scientific Research Publishing Inc. This work is licensed under the Creative Commons Attribution International License (CC BY 4.0).

http://creativecommons.org/licenses/by/4.0/ (c) (i) Open Access

\begin{abstract}
Background: Approximately $11 \%$ of cancer cases are diagnosed in people of childbearing age. Borderline ovarian tumors (BOTs) make up 10\% - 15\% of all ovarian epithelial malignancies. More than one-third of all BOTs occur in women under 40 years of age. Maintaining the fertility of cured patients is the common goal of both oncologists and reproductologists. Aim: Giving young women diagnosed with a prognostically worse type of BOT and after bilateral adnexectomy the possibility to have their genetically own children by the method of ex vivo oocyte collection. Case Presentation: A 34-year-old nulligravid woman with BOT underwent right laparoscopic salpingo-oophorectomy. Histologically, a serious borderline tumor with a micropapillary pattern and a tumor locus on the ovarian surface were found. Due to histopathology, the oncologist recommended re-staging surgery: laparotomy, left salpingooophorectomy, omentectomy and hysterectomy. The patient refused a hysterectomy as she was planning to get pregnant with her partner. To maintain her fertility, controlled hormonal hyperstimulation and ex vivo aspiration of follicles from the ovary after salpingo-oophorectomy was performed. Ex vivo follicle expiration yielded 10 oocytes. 9 mature oocytes were fertilized by ICSI. The 6 embryos of the highest quality were individually frozen by vitrification. Cryoembryotransfer will be scheduled with the consent of the oncologist. Conclusion: This method is suitable for young women with BOT after bilateral salpingo-oophorectomy in whom ex vivo oocyte collection prevents possible leakage of tumor cells into the abdominal cavity, unlike during the conventional in vivo collection prior to surgery.
\end{abstract}




\section{Keywords}

Borderline Ovarian Tumor, Radical Surgery, In Vitro Oocyte Collection, Maintain Fertility, Oncofertility

\section{Introduction}

Every year, more than 200,000 malignancies are diagnosed in women and men under the age of 45 . As a result of new therapeutic techniques, the number of people of childbearing age who have been successfully treated for cancer is growing. In an effort to help the young people, who were successfully treated for cancer, to have their genetically own children, the medical subspecialization "oncofertility" has been created. It is an interdisciplinary collaboration between oncologists and specialists in reproductive medicine and reproductive biology [1]. Current fertility options for young women and girls include 1) cryopreservation and subsequent ovarian tissue transplantation and 2) cryopreservation of ovarian tissue and subsequent in vitro oocytes maturation and freezing oocytes or embryos for later cryoembryotransfer [2] [3]. The condition is that the used techniques for preservation of reproduction do not adversely affect cancer treatment [4] [5].

Borderline ovarian tumors (BOTs) account for 10\% - 15\% of all ovarian epithelial malignancies. Their annual prevalence in Europe is approximately 4.8/100,000 new cases. More than 1/3 of all BOTs are found in women under the age of 40 [6] [7]. BOTs are characterized by higher epithelial proliferation and more variable nuclear atypia than benign lesions, but unlike carcinomas, they do not have stromal invasion [8]. 53\% - 65\% are serious BOTs, approximately $32 \%$ are mucinous, the rests are endometrial, clear cell and Brenner tumors. Surgery is the primary treatment for BOTs, as in the treatment of malignant ovarian cancer, hysterectomy, bilateral salpingo-oophorectomy, omentectomy, peritoneal lavage with multiple biopsies, and appendectomy (in the case of mucinous BOT) are standard procedures. For women who want to have children, doctors try to turn radical surgery into a conservative procedure with preservation of the uterus and ovary. Histopathological findings are decisive for the choice of surgical procedure. Micropapillary serous BOTs have a worse prognosis with the more frequent bilateral occurrence and more frequent invasive peritoneal implants. Therefore, bilateral salpingo-oophorectomy, omentectomy, lavage, and multiple peritoneal biopsies are recommended in a conservative procedure. The uterus is preserved for the planned conception. Regular ultrasound follow-up is essential until hysterectomy [9].

In this case report, we draw attention to the possibility of collecting mature oocytes, after controlled ovarian hyperstimulation ( $\mathrm{COH})$, ex vivo. The method is suitable for young women in whom bilateral salpingo-oophorectomy for BOTs is indicated and where ex vivo collection of oocytes prevents possible leakage of 
tumor cells into the abdominal cavity during conventional in vivo collection before surgery [10].

\section{Case Report}

A 34-year-old nulligravid woman without relevant medical history and unaware of any family history of ovarian or breast cancer plans to get pregnant with her partner.

Physical examination and ultrasound discovered a suspected borderline ovarian tumor on the right ovary.

Ultrasound with the vaginal probe: Uterus in AVF, normal size, symmetrical, endometrium triple line up to $6 \mathrm{~mm}$. Right ovary: Total size $45 \times 29 \mathrm{~mm}$ with small follicles and one follicle with a diameter of $26 \mathrm{~mm}$ with numerous papillary prominences exceeding $7 \mathrm{~mm}$, solid component $19 \times 9 \mathrm{~mm}$, relatively rich vascular supply of the solid component. Left ovary is of normal appearance. Abdominal cavity is without free fluid.

The tumor markers CA 125 and HE-4 were negative.

Laparoscopically right-sided salpingo-oophorectomy using endobag, biopsy of peritoneum and aspiration of effusion for cytology was performed (Figure 1, Figure 2).

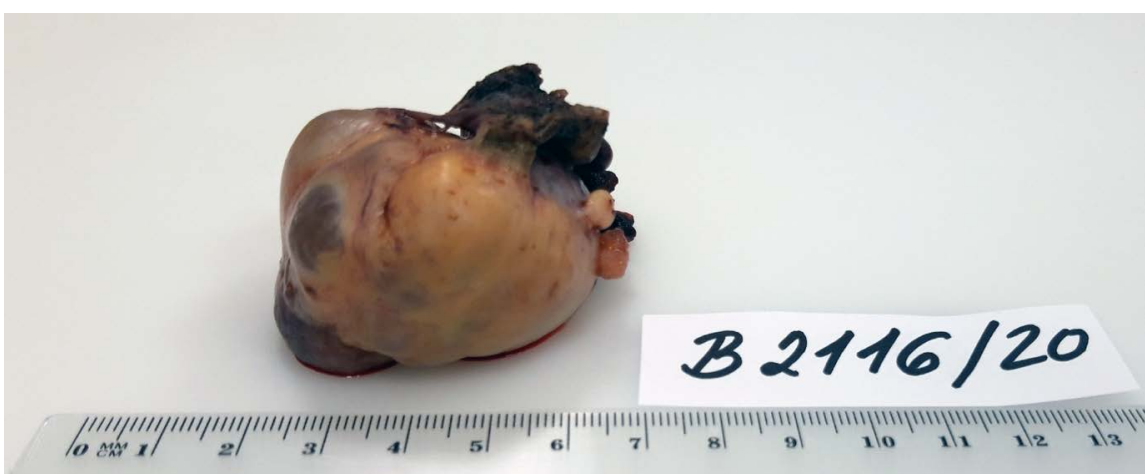

Figure 1. Right ovary with papillary prominences and a tumor locus on the ovarian surface.

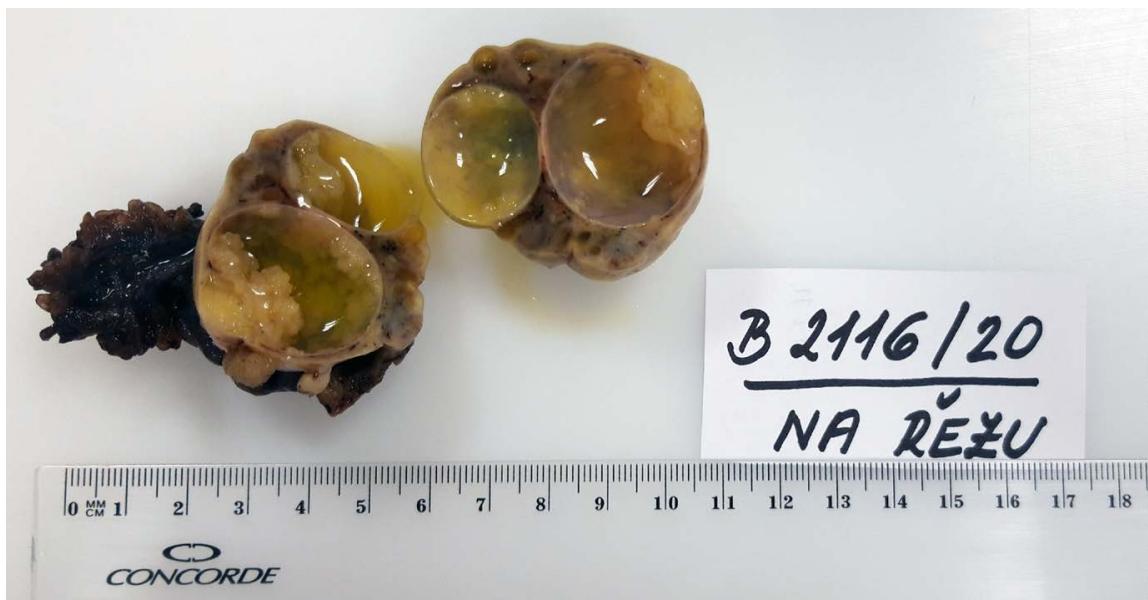

Figure 2. Cross section of the right ovary. 
Histology: serous borderline tumor, locally with micropapillary structure and a tumor locus on the ovarian surface, with fibrous adhesions in the vicinity. Peritoneal effusion and excision without signs of tumor (Figure 3, Figure 4).

Due to the histopathological finding and postoperative staging (pT1C2), the oncologist recommended a re-staging surgery: laparotomically performed left salpingo-oophorectomy, omentectomy, appendectomy (due to adhesions to right ovary), and hysterectomy.

The patient opted against hysterectomy, as she wished to keep the uterus and thus retain the possibility of getting pregnant.

Her AMH after surgery was $3.91 \mathrm{ng} / \mathrm{ml}$.

Examination of the partner's spermiogram according to WHO criteria showed normal sperm count, morphology, and movement.

The oncologist did not want to delay the surgery unnecessarily. He consulted a reproductologist on the possibility of maintaining the patient's fertility.

The options given were:

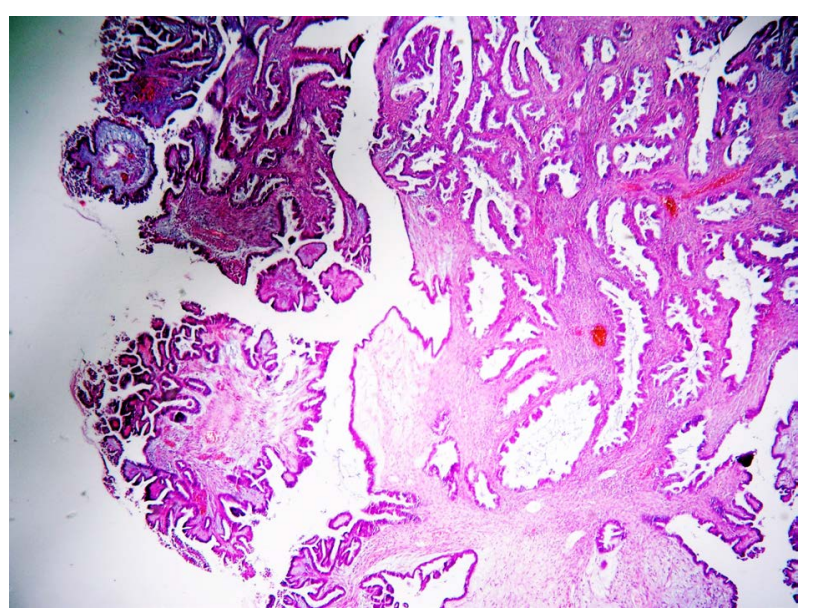

Figure 3. Serous borderline tumor shows focal advanced micropapillary pattern.



Figure 4. Serous borderline tumor and a part of ovary with primary follicles, antral follicle in the right corner. 
1) Freezing of ovarian tissue for later transplantation, but with a risk of clogging the tumor cells;

2) Freezing of ovarian tissue and maturation of oocyte in vitro, but this method is still not very successful and considering the age of the patient (34 years old);

3) $\mathrm{COH}$ with recombinant FSH (rec.FSH) and oocyte collection prior to salpingo-oophorectomy; however, clogging of tumor cells in the peritoneal cavity during follicular puncture cannot be ruled out;

4) $\mathrm{COH}$ rec.FSH, salpingo-oophorectomy and ex vivo follicle aspiration, followed by oocyte freezing or oocyte fertilization by ICSI and embryo freezing; however, we have no practical experience with ex vivo aspiration of oocytes;

5) Donated oocyte, surrogate mother.

The possibilities of maintaining fertility were explained to the patient and her partner and they had the opportunity to decide which option to choose until the next day.

The partners decided to collect oocytes in vitro, fertilize them with sperm using the ICSI technique and freeze the embryos.

On day 14 of the menstrual cycle after ultrasound examination (anteverted uterus, endometrium $5 \mathrm{~mm}$, ovary on the left with follicles up to $5 \mathrm{~mm}$, on the right side is the absence of ovary-patient after salpingo-oophorectomy, rectouterine pouch without free fluid) we started $\mathrm{COH}$ with rec.FSH (Puregon pen $175 \mathrm{IU} / 1)$.

Day 6 of stimulation, with follicles up to $12 \mathrm{~mm}$, we added $\mathrm{GnRH}$ antagonist (Cetrotide $250 \mathrm{MCG} / 0.5 \mathrm{ml}$ ).

Day 11 of stimulation, endometrium $10 / 2 \mathrm{~mm}$, left ovary with about 15 follicles 14 - $20 \mathrm{~mm}$ in diameter, HCG was applied for ovulation timing (Ovitrelle $250 \mathrm{MCG} / 0.5 \mathrm{ml})$.

A laparotomy was performed on day 13 from the start of stimulation and 35 hours after Ovitrelle administration.

The left ovary with the fallopian tube was gently removed and placed in a sterile container with saline solution (stimulated ovary measuring about $8 \times 8 \mathrm{~cm}$ ), after which omentectomy and appendectomy were performed.

The ovary with the fallopian tube was immediately transferred to the IVF operation room, which is connected by a window to the embryological laboratory. Two minutes after surgical salpingo-oophorectomy ex vivo aspiration of follicles began.

Suction tubes from the COOK Ovum aspiration needle kit (K-OSN-1730B-90) were used to aspirate the follicles ex vivo. (We cut the original $17 \mathrm{GA} / 30$ $\mathrm{cm}$ needle from the tubing and attached a pink $1.2 \mathrm{~mm}$ in length $\times 40 \mathrm{~mm}$ in diameter intramuscular needle to the original tubing using a GAMA infusion cap with a rubber stopper.). Prior to aspirating the follicles, a tightness test was performed.

Suction of the follicular fluid was performed with the same vacuum as in the 
classical transvaginal oocyte collection, i.e. $130 \mathrm{mmHG}$ (COOK aspirator). Immediately after aspiration of the follicular fluid, each follicle was rinsed once with saline.

Follicular fluid was passed in tubes to embryologists to search for oocytes (standard procedure).

10 oocytes (9 mature, 1 immature) were obtained.

The whole process from removing the left ovary to the completion of oocyte aspiration in vitro lasted for 10 minutes.

After oocyte collection, the ovary and the fallopian tube were transferred to a histological laboratory for a histopathological examination (Figures 5-9).

All 9 mature oocytes were fertilized by ICSI. The 4 highest quality embryos were separately frozen by vitrification 96 hours after fertilization. 3 in the morula stage, 1 in the cavitating blastocyst stage. The other 2 embryos were separately frozen by vitrification 120 hours after fertilization. One is in the blastocyst stage, the other is in the morula stage.

The patient was discharged on the third day after the surgery.

The result of histopathology: The left fallopian tube, ovary, omentum and appendix showed no signs of a serious borderline tumor or other malignancy. Chronic appendicitis was diagnosed.

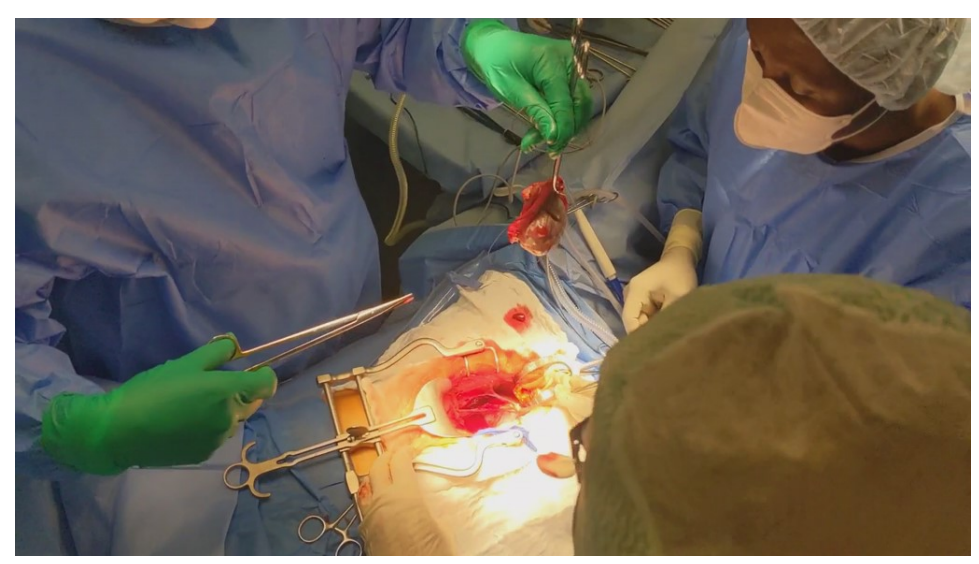

Figure 5. Left salpingo-oophorectomy.

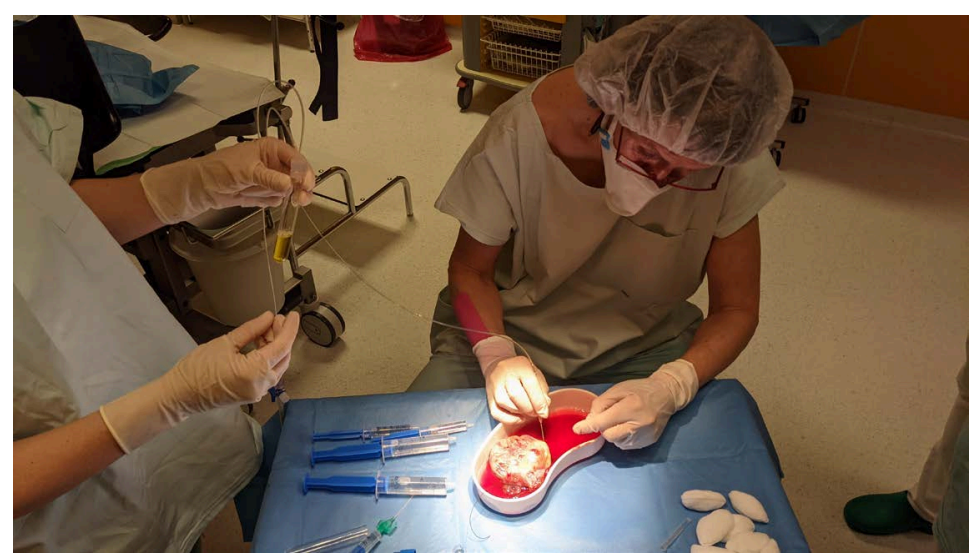

Figure 6. Follicle aspiration ex vivo. 


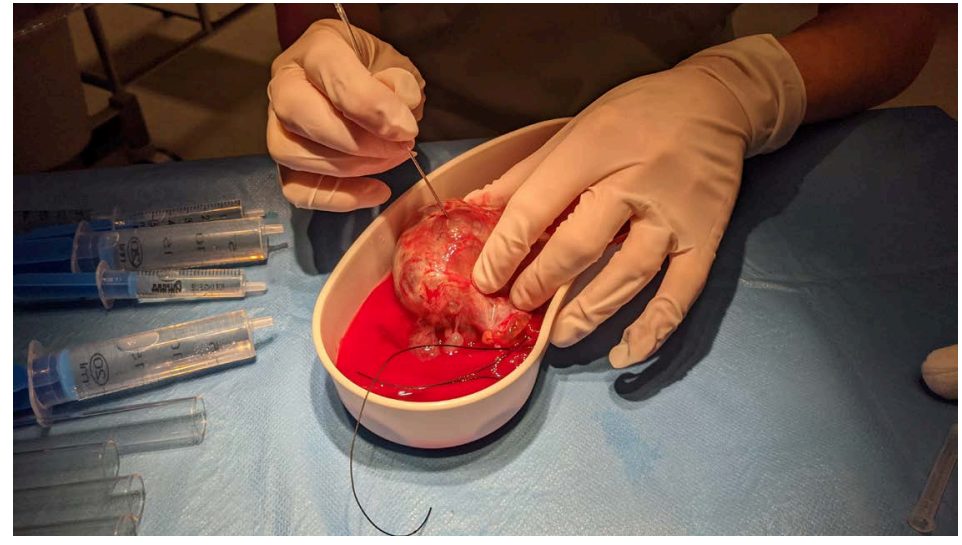

Figure 7. Aspiration of individual follicles.

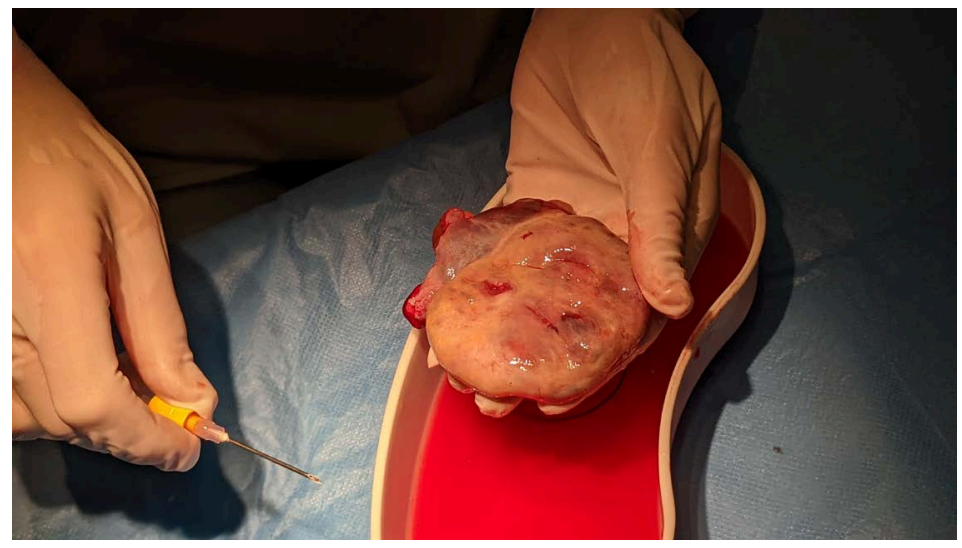

Figure 8. Control of the ovary after aspiration and rinsing of all follicles.

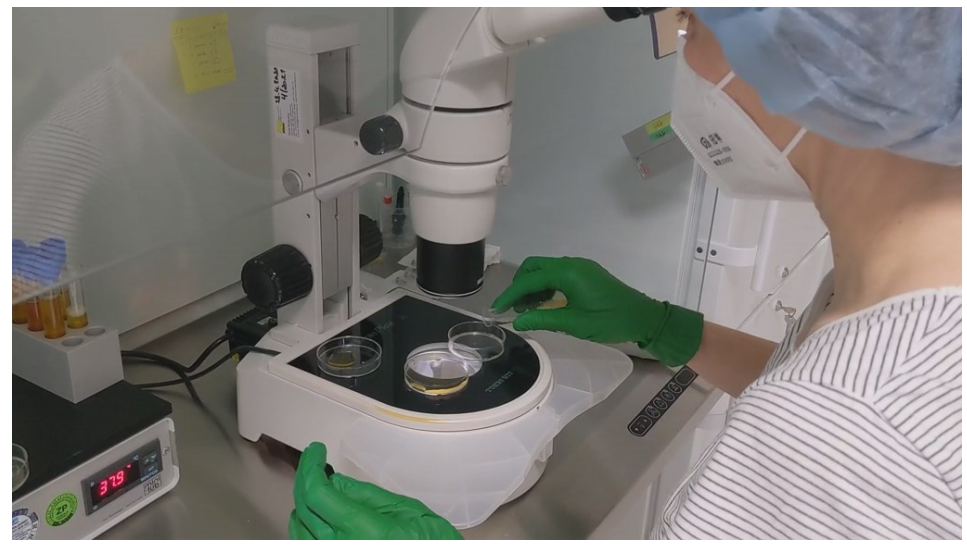

Figure 9. Searching for oocytes in follicular fluid under a microscope in embryological laboratory.

With the consent of an oncologist, we plan to perform cryoembryotransfer at the earliest convenience.

\section{Discussion}

Radical surgery is recommended as the primary treatment for both borderline ovarian tumor and ovarian cancer. However, patients with BOTs are significant- 
ly younger than patients with invasive ovarian cancer. $27 \%$ - $54 \%$ are under the age of 40 and want to retain fertility [9]. Therefore, the treatment of BOTs has changed from radical surgery (hysterectomy and bilateral salpingo-oophorectomy) to a more conservative one; however, there is still no consensus on the surgical solution. BOTs are characterized by a high recurrence rate after conservative surgery. The recurrence rate on the remaining ovary is about $10 \%$ in patients with unilateral stage I borderline ovarian tumors who undergo unilateral salpingooophorectomy. The risk of recurrence is increased to $30 \%-40 \%$ if cystectomy is performed. However, patients are advised to get pregnant as soon as possible.

Because the chance of spontaneous conception after conservative BOT surgery is reduced, in vitro fertilization (IVF) is a good choice, as it significantly increases the likelihood of pregnancy.

$\mathrm{COH}$ is not a risk factor in patients in the early stages of BOTs. IVF will also allow the freezing of oocytes and embryos for later cryoembryotransfer [11] [12].

Cryopreservation of ovarian tissue for later transplantation is not recommended, as there is a potential risk of retransplanted tumor cells. A more convenient method is to obtain immature oocytes from frozen ovarian tissue and mature them in vitro, but this method of maintaining fertility is not yet routinely performed [13].

Fatemi reported the first case of ex vivo acquisition of mature oocytes after ovarian stimulation [10]. Our experience has confirmed that after $\mathrm{COH}$, which can be initiated at any time during the menstrual cycle [14], mature oocytes can be obtained, after classical induction of ovulation by follicular aspiration ex vivo, without the risk of cancer cell spillage associated with the standard transvaginal oocyte collection. Monitoring of patients after BOT surgery (physical examination, ultrasonography and control of tumor markers) is recommended for 10 years, at least once a year [13].

\section{Conclusion}

Ex vivo oocyte collection provides a new possible strategy for fertility preservation in patients with the prognostically worse types of BOTs, in whom bilateral adnexectomy is required. Ex vivo oocyte collection prevents possible leakage of tumor cells into the abdominal cavity, unlike during conventional in vivo oocyte collection prior to surgery.

\section{Acknowledgements}

We thank Jaroslav Feyereisl, director of the Institute for the Care of Mother and Child, who enables us to perform medicine at the highest level, including oncofertility.

\section{Authors' Contributions}

J.R. designed and performed ex vivo oocyte collection and prepared the article, P.S. performed the oncological operation and approved the procedure for main- 
taining fertility, L.P. figured out how to adapt the suction system for oocyte collection $e x$ vivo and assisted in oocyte puncture, B.K. looked for oocytes after $e x$ vivo aspiration of follicles, J.P. shot the photo documentation, J.R. performed the fertilization of oocytes using the ICSI technique, R.V. and K.D. controlled the development of embryos and ensured their freezing, J.R. helped with the elaboration of the article and carried out linguistic proofreading, P.D. and M.B. conducted a literature search, J.F. approved the final version of the article.

\section{Conflicts of Interest}

The authors declare no conflicts of interest regarding the publication of this paper.

\section{References}

[1] Woodruff, T.K. (2015) Oncofertility: A Grand Collaboration between Reproductive Medicine and Oncology. Reproduction, 150, 1-10.

https://doi.org/10.1530/REP-15-0163

[2] Dursan, P., Dogan, N. and Ayhan, A. (2014) Oncofertility for Gynecologic and Non-Gynecologic Cancers: Fertility Sparing in Young Women of Reproductive Age. Critical Reviews in Oncology/ Hematology, 92, 258-267. https://doi.org/10.1016/j.critrevonc.2014.07.001

[3] Demeestere, I., Simon, I., Dedeken, I., et al. (2015) Live Birth after Autograft of Ovarian Tissue Cryopreserved During Childhood. Human Reproduction, 30, $2107-$ 2109. https://doi.org/10.1093/humrep/dev128

[4] Woodruff, T.K. (2010) The Oncofertility Consortium-Addressing Fertility in Young People with Cancer. Nature Reviews Clinical Oncology, 7, 466-475. https://doi.org/10.1038/nrclinonc.2010.81

[5] Rezacova, J., et al. (2018) Oncofertility: Reproductive Medicine, Current Possibilities in Assisted Reproduction. Monograph, 280-289.

[6] Trope, C.G., et al. (2000) Surgery for Borderline Tumor of the Ovary. Seminars in Surgical Oncology, 19, 69-75. https://doi.org/10.1002/1098-2388(200007/08)19:1<69::AID-SSU11>3.0.CO;2-E

[7] Morice, P., et al. (2012) Borderline Ovarian Tumor: Pathological Diagnostic Dilemma and Risk Factor for Invasive or Lethal Recurrence. The Lancet Oncology, 13, 103-115. https://doi.org/10.1016/S1470-2045(11)70288-1

[8] Trillsch, F., Mahner, S., Ruetzel, J., et al. (2010) Clinical Management of Borderline Ovarian Tumors. Expert Review of Anticancer Therapy, 10, 1115-1124.

https://doi.org/10.1586/era.10.90

[9] Alvarez, R.M. and Vazquez-Vicente, D. (2015) Fertility Sparing Treatment in Borderline Ovarian Tumours. Encancer, 9, Article ID: 507. https://doi.org/10.3332/ecancer.2015.507

[10] Fatemi, H.M., Kyrou, D., Al-Azemi, M., et al. (2011) Ex-vivo Oocyte Retrieval for Fertility Preservation. Fertility and Sterility, 95, 15-17. https://doi.org/10.1016/j.fertnstert.2010.11.023

[11] Beiner, M.E., Gotlieb, W.H., Davidson, B. et al. (2001) Infertility Treatment after Conservative Management of Borderline Ovarian Tumours. Cancer, 92, 320-325. https://doi.org/10.1002/1097-0142(20010715)92:2<320::AID-CNCR1325>3.0.CO;2-G 
[12] Facouliotis, S.J., Davis, O., Schattman, G., et al. (2004) Safety and Efficacy Treatment after Conservative Management of Borderline Ovarian Tumours: A Preliminary Report. Fertility and Sterility, 82, 568-572.

https://doi.org/10.1016/j.fertnstert.2004.02.114

[13] Maramai, M., Barra, F., Menada, M.V., et al. (2020) Borderline Ovarian Tumours: Management in the Era of Fertility-Sparing. Encancer, 14, 1031.

https://doi.org/10.3332/ecancer.2020.1031

[14] Kuang, Y., Hong, Q., Chen, Q., et al. (2017) Luteal-Phase Ovarian Stimulation is Feasible for Producing Competent Oocytes in Women Undergoing in Vitro Fertilization/Intracytoplasmatic Sperm Injection Treatment, with Optimal Pregnancy Outcomes in Frozen-Thawed Embryo Transfer Cycles. Fertility and Sterility, 101, 105-111. https://doi.org/10.1016/j.fertnstert.2013.09.007 